\title{
Pediatric tibial shaft and distal metaphyseal fractures
}

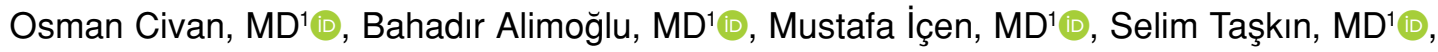 \\ Ali Cavit, $M D^{2}$, Yetkin Söyüncü, $M^{1}{ }^{1 D}$, Mustafa Ürgüden, $M^{1}{ }^{1}$ \\ ${ }^{1}$ Department of Orthopedics and Traumatology, Medicine Faculty of Akdeniz University, Antalya, Turkey \\ ${ }^{2}$ Department of Orthopedics and Traumatology, Medicine Faculty of Uludağ University, Bursa, Turkey
}

Pediatric trauma has some different points from adults and the incidence is increasing over the years. Nearly one of every four children needs medical care due to an accidental injury in the United States. ${ }^{[1]}$ Tibial shaft fractures are the third most common long bone fracture type in children after fractures of radius-ulna and femur; and the second most common reason for hospitalization after femur fractures. ${ }^{[2-4]}$

Pediatric tibial fractures (PTFs) are mostly treated by closed reduction and casting (CRC). Sometimes, the family of a three- or four-year-old child with spiral and non-displaced fracture of the tibial shaft, Toddler's fracture, may not prefer to come to the hospital urgently, since children may have little pain, and just walk with antalgic full weight bearing. If the family is questioned about the patients' history, they may report unwillingness of the patient to bear weight. ${ }^{[5]}$

Received: April 29, 2020

Accepted: June 18, 2020

Published online: September 11, 2020

Correspondence: Osman Civan, MD. Akdeniz Üniversitesi Tıp Fakültesi Ortopedi ve Travmatoloji Anabilim Dalı, 07070 Kampus, Antalya, Türkiye.

E-mail: civanosman@gmail.com

Doi: $10.5606 /$ ehc. 2020.75764

Citation: Civan $O$, Alimoğlu B, İçen M, Tasskın S, Cavi† A, Söyüncü $Y$, et al. Pediatric tibial shaft and distal metaphyseal fractures. Jt Dis Relat Surg 2020;31(3):532-540

(2020 All right reserved by the Turkish Joint Diseases Foundation

This is an open access article under the terms of the Creative Commons Attribution-NonCommercial License, which permits use, distribution and reproduction in any medium, provided the original work is properly cited and is not used for commercial purposes (http://creativecommons.org/licenses/by-nc/4.0/).

\section{ABSTRACT}

Objectives: This study aims to investigate the effects of associated factors like age, fracture level, accompanying fibular fractures and wound condition on healing by determining and comparing union scores of pediatric tibial fractures (PTFs).

Patients and methods: Forty-five patients with 46 PTFs (32 males, 13 females; mean age 9.5 years; range 2 to 16 years) who were treated by closed reduction and casting or operated between January 2016 and January 2019 were retrospectively evaluated. The union scores were evaluated at the end of fourth, sixth and eighth weeks and compared to each other. Effects of the age, associated fibular fractures, wound condition, fracture level and treatment type to union score were analyzed.

Results: Twenty-eight (60.9\%) out of 46 PTFs had associated fibular fractures and $18(39.1 \%)$ did not. Motor vehicle accident was the most frequent etiologic factor $(47.8 \%)$. Thirty-four out of 46 fractures were closed tibial fractures $(73.9 \%)$ and 12 had open wound $(26.1 \%)$. There was a negative correlation between age and the union scores $(\mathrm{p}<0.001$ for each week). No significant difference was observed between the union score of diaphyseal and metaphyseal fractures at the fourth, sixth, and eighth weeks. The union scores of each week were higher in the conservative group compared to operative group ( $\mathrm{p}<0.001$ for each week). Associated fibular fracture group had lower union scores compared to isolated tibial fracture group at fourth, sixth and eighth weeks. Likewise, the union scores of the open fracture group were lower than the closed fracture group ( $\mathrm{p}<0.05$ for each week).

Conclusion: Associated fibular fractures, open fractures and aging negatively affect union scores of PTFs. Attention should be paid, particularly in these conditions, during the selection of the operation type and the follow-up period of PTFs.

Keywords: Non-union, pediatric fractures, pediatric tibial fractures, tibial fractures, union.

Injury type, age, fracture type, the amount of displacement, soft tissue condition, associated fibular fracture and weight may affect the choice of treatment. The interval of the management is wide and may change from casting to the multiaxial, circular external fixator, or osteosynthesis with open reduction and plating. As the child grows, the force 
required to break the tibia increases and the treatment type changes from casting to operation. ${ }^{[5-8]}$

There have been several types of methods for assessing the union rate while there is still no gold standard method. ${ }^{[9,10]}$ In this study, we aimed to investigate the effects of associated factors like age, fracture level, accompanying fibular fractures and wound condition on healing by determining and comparing union scores of PTF.

\section{PATIENTS AND METHODS}

In this study, we retrospectively evaluated 58 patients with PTFs who required closed reduction and were treated in the operating room under fluoroscopy at Department of Orthopedics and Traumatology of Akdeniz University, Faculty of Medicine between January 2016 and January 2019. Patients with head trauma, proximal metaphyseal tibial fractures, fractures extending to the ankle joint and knee joint or extending from shaft to more than $2 \mathrm{~cm}$ below to the tibial tuberosity, fractures more proximal from $2 \mathrm{~cm}$ below to the tibial tuberosity, epiphyseal fractures, Toddler's fractures, greenstick fractures, torus fractures, bowing, and patients who could not be followed-up for at least one year were excluded. Forty-five patients with 46 PTFs (32 males, 13 females; mean age 9.5 years; range 2 to 16 years) met the inclusion criteria. The study was conducted in accordance with the principles of the Declaration of Helsinki.

For retrospective evaluation, the hospital archiving system (Mia-Med version 1.0.1.2808, Mia Technology A.Ş, Ankara, Türkiye) and Picture Archiving and Communication System (PACS; Version 21.1, February 2019; Sectra Workstation, Linköping-Sweden) were used for collecting demographic information, examination reports of the emergency consultation and additional problems, procedures applied during followup periods and detailed operation reports. The association between age, sex, side, fracture type, soft tissue condition, fibular fracture and fracture healing were evaluated.

In patients younger than eight years of age, 10 degrees of angulation, $10 \mathrm{~mm}$ shortening and apposition more than $50 \%$ were accepted. If the patient was older than eight years of age, angulation less than five degrees was accepted as proper in the present study. ${ }^{[7]}$ Patients with fractures in which these criteria could not be provided, open fractures and multiple-trauma were operated. ${ }^{[7,11]}$ Fractures that could not meet the acceptable criteria, which were listed above, when they were accepted to the emergency department or fractures that could not meet the acceptable criteria without reduction under fluoroscopy were included in the study.

Fracture type was determined and classified by three orthopedic surgeons according to the Arbeitsgemeinschaft für Osteosynthesefragen (AO)Pediatric Comprehensive Classification of Long Bone Fractures (PCCF) classification system. ${ }^{[12]}$ Due to the insufficiency of the number of each group, fractures were also classified as distal and shaft. We classified fractures according to the soft tissue condition by using Gustilo Anderson (GA) classification. ${ }^{[13]}$ Treatment type for each fracture and the association between union score and additional problems were analyzed.

For determining the union score, we used the Radiological Union Score of Tibia (RUST) that was described by Whelan et al ${ }^{[9]}$ to define the union score of tibial fractures treated by intramedullary nail. RUST was calculated by three orthopedic surgeons. If the calculated value was more than 0.5 , it was rounded up to the next higher number and if it was less than 0.5, it was rounded up to the next lesser number. Union scores of fourth, sixth and eighth weeks were recorded.

The effect of associated fibular fracture on the union score was one of the other mainly investigated subjects. The AO-PCCF includes different classification types considering whether fibula is fractured or not. We included tibial fractures in the same group if they were at the same level regardless of whether fibula was fractured or not to determine the effect of fibular fracture on the union score.

Additional operation and additional postoperative problems were determined for each patient. The relationships between fracture type and these subgroups were analyzed.

We applied the same procedures for all closed fractures. We used cefazolin for preoperative prophylaxis and continued three times until the end of the first 24 hours postoperatively. We applied debridement and obtained cultures if the wound was in bad condition regardless of the GA classification and used broad-spectrum antibiotics.

Postoperative protocols changed according to the applied treatment type. Patients were called for second-, fourth-, sixth- and eighth-week controls as a routine follow-up procedure. The control time changed according to the treatment type and visible callus. Weight bearing was allowed when the callus was visible in the radiography. 


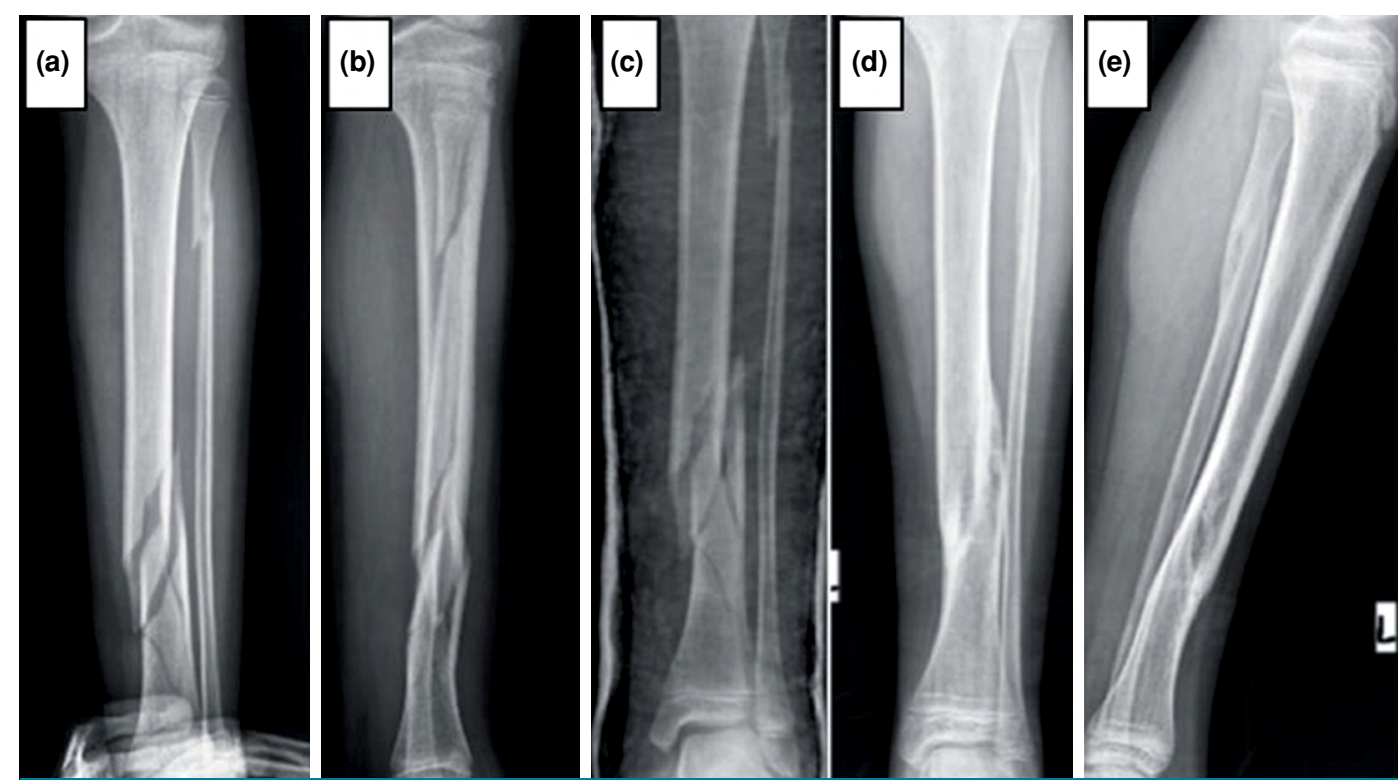

FIGURE 1. A 13-year-old male patient's left tibial and associated fibular fracture after a motor vehicle accident (AO-PCCF 42-D/5.2). (a) Preoperative anteroposterior X-ray. (b) Preoperative lateral view. (c) Anteroposterior view after closed reduction and casting. (d) First-year anteroposterior radiography, no malalignment after remodeling. (e) First-year lateral radiography, no malalignment after remodeling. AO-PCCF 42-D/5.2: Arbeitsgemeinschaft für Osteosynthesefragen Pediatric Comprehensive Classification of Long Bone Fractures type 42-D/5.2.

\section{Statistical analysis}

Pearson's chi-square and/or Fisher's exact tests were used for the analysis of the categorical data. Shapiro-Wilk test was used for testing normality. Mann-Whitney $U$ test was used for those which did not fit the normal distribution. Kruskal-Wallis test was used for triple comparison. BonferroniDunn procedure was used for pairwise comparison. The relationships between continuous variables were analyzed by Spearman's correlation analysis. All statistical analyses were conducted by the IBM SPSS version 23.0 software (IBM Corp., Armonk, NY, USA) and $p$ values less than 0.05 were considered statistically significant.

\section{RESULTS}

The affected side was right for $22(48.9 \%)$ patients, left for $22(48.9 \%)$ patients and bilateral for one $(2.2 \%)$
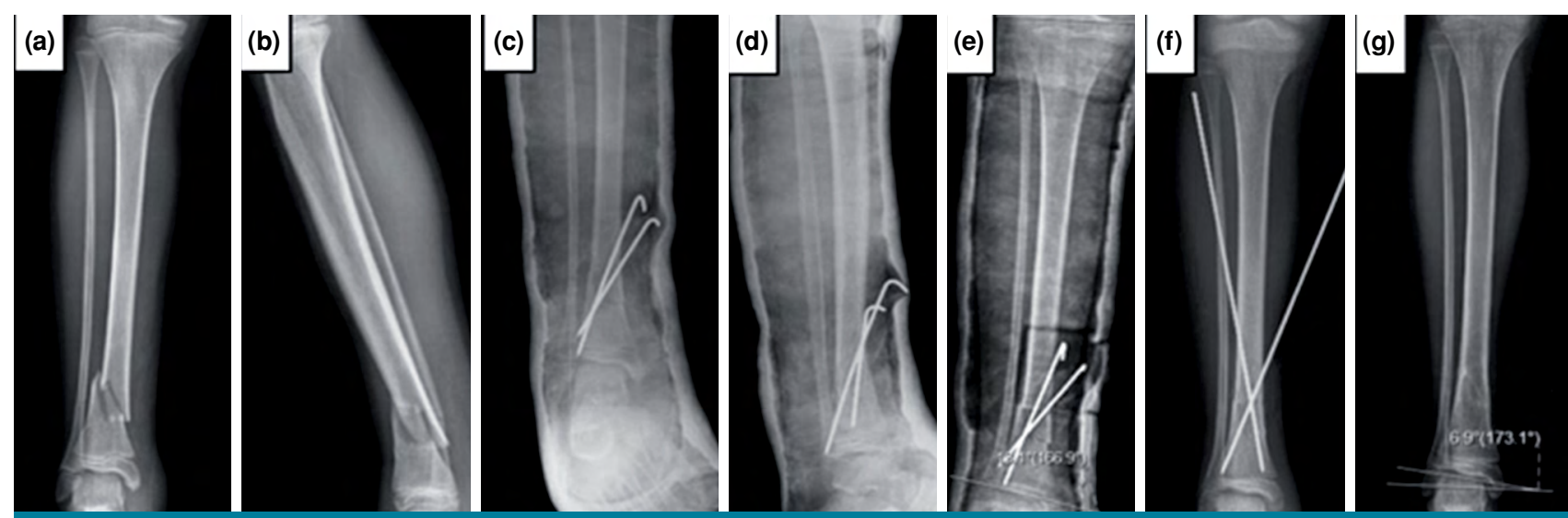

FIGURE 2. A 10-year-old male patient's right tibial and associated fibular fracture after a motor vehicle accident (AO-PCCF 43-M/3.1). (a) Preoperative anteroposterior view. (b) Preoperative lateral view. (c) Anteroposterior view after first operation. (d) Lateral view after first operation. (e) Thirteen degrees valgus malalignment at fourth week. (f) Revision surgery: Closed reduction + pinning and casting again. (g) First-year anteroposterior view, perfect union score and $6.9^{\circ}$ valgus alignment.

AO-PCCF 43-M/3.1: Arbeitsgemeinschaft für Osteosynthesefragen Pediatric Comprehensive Classification of Long Bone Fractures type 43-M/3.1. 
patient. Four patients had co-existing fractures (two femur, one humerus, one tibia fractures) and three patients had concomitant pathologies as follows: one ruptured spleen (open reduction and plating) and two pneumothoraces (open reduction and plating and titanium elastic nail). Twenty-eight $(60.9 \%)$ out of 46 tibial fractures had associated fibular fractures and $18(39.1 \%)$ did not. Motor vehicle accident (auto, pedestrian or bicycle) was the most frequent etiologic factor (47.8\%). Thirtyfour out of 46 fractures were closed tibial fractures (73.9\%) and 12 had open fractures (26.1\%). Two were GA classification type $1(4.3 \%)$, seven were type $2(15.2 \%)$, one was type 3A $(2.2 \%)$ and two were type 3C (4.3\%). Applied treatment types were as follows: CRC (Figure 1) for 17 fractures $(37 \%)$, closed reduction + pinning and casting (Figure 2) for six fractures (13\%), titanium elastic nail for eight fractures (17.4\%), open reduction and plating (Figure 3) for five fractures (10.9\%), external fixator (Figure 4) for nine fractures (19.6\%) and rigid intramedullary nail (Figure 5) for one fracture $(2.2 \%)$. After these treatments, 23 fractures (50\%) had some problems as follows: 16 fractures had maximum union score of 12 after eight weeks, four fractures had a valgus deformity, one fracture had synostosis (Figure 5), and two had wound problems and infection. Four fractures (9\%) of these 23 needed more interventions and these were due to valgus deformity $\left(>10^{\circ}\right)$ for one patient (Figure 2), bone defect for one patient and delayed union for two patients. The demographic data of the patients and the informative details about the fractures are listed in Table I and the mean union score for each week is listed in Table II.

The mean age of the male population (10.3 years) was higher than the female (7.5 years). There was a negative correlation between age and the union scores $(\mathrm{p}<0.001 / \mathrm{r}=-0.778 ; \mathrm{p}<0.001 / \mathrm{r}=-0.770 ; \mathrm{p}<0.001 /$ $\mathrm{r}=-0.758$, respectively for each week). Although mean age of the patients injured by motor vehicle accident (auto, pedestrian or bicycle) was 11.2 years, the type of trauma was not statistically related to the age in pairwise comparisons (falling from height or stairs is group 1, motor vehicle accident is group 2 and the others are group 3; group 1-2: $\mathrm{p}=0.054$, group 1-3: $\mathrm{p}=1.000$, group 2-3: $\mathrm{p}=0.321$ ).

The union scores for each week did not change statistically according to sex $(\mathrm{p}=0.338 ; \mathrm{p}=0.375$; $\mathrm{p}=0.227$, respectively for fourth, sixth and eighth weeks). The delayed union rate of males was higher

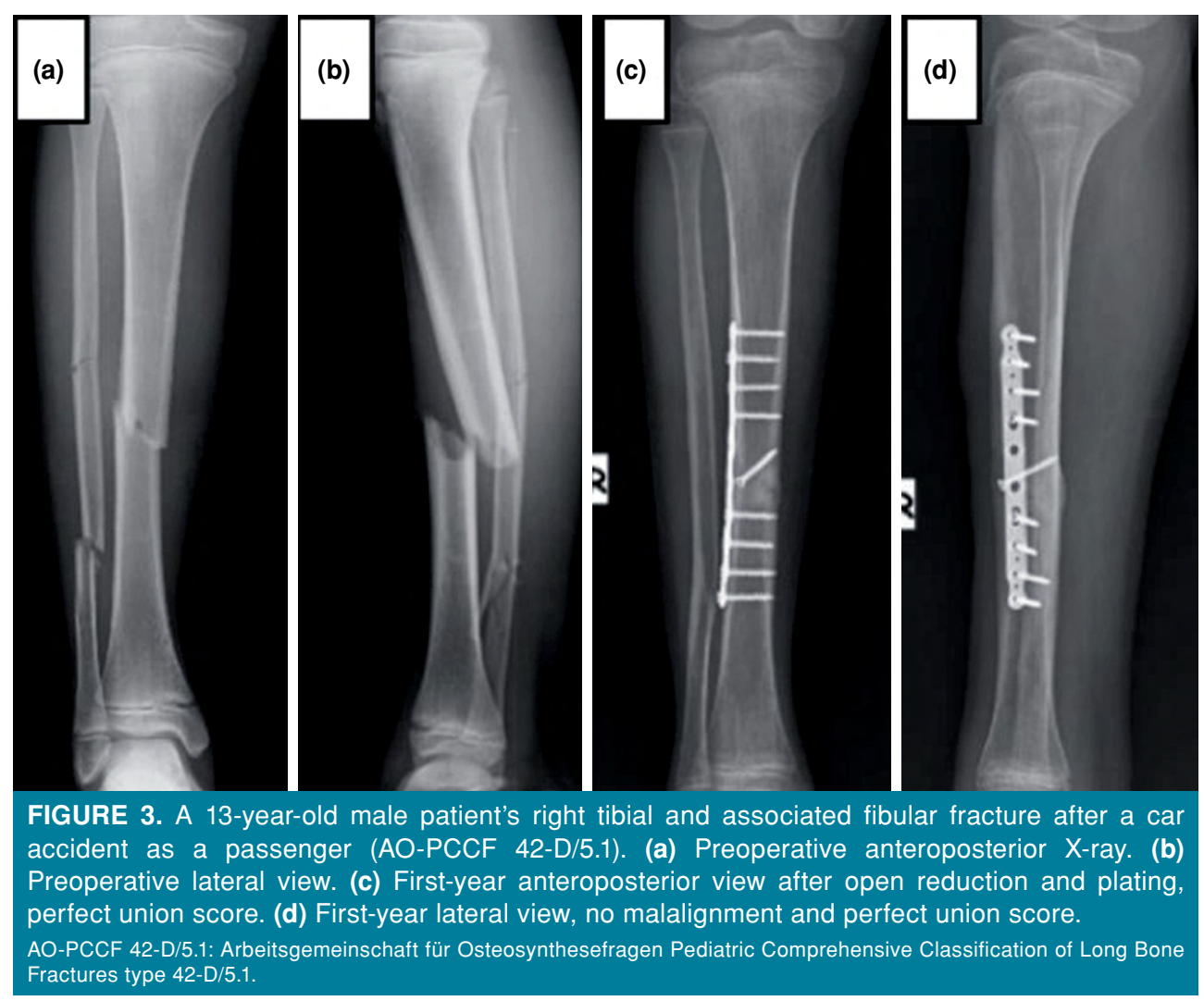



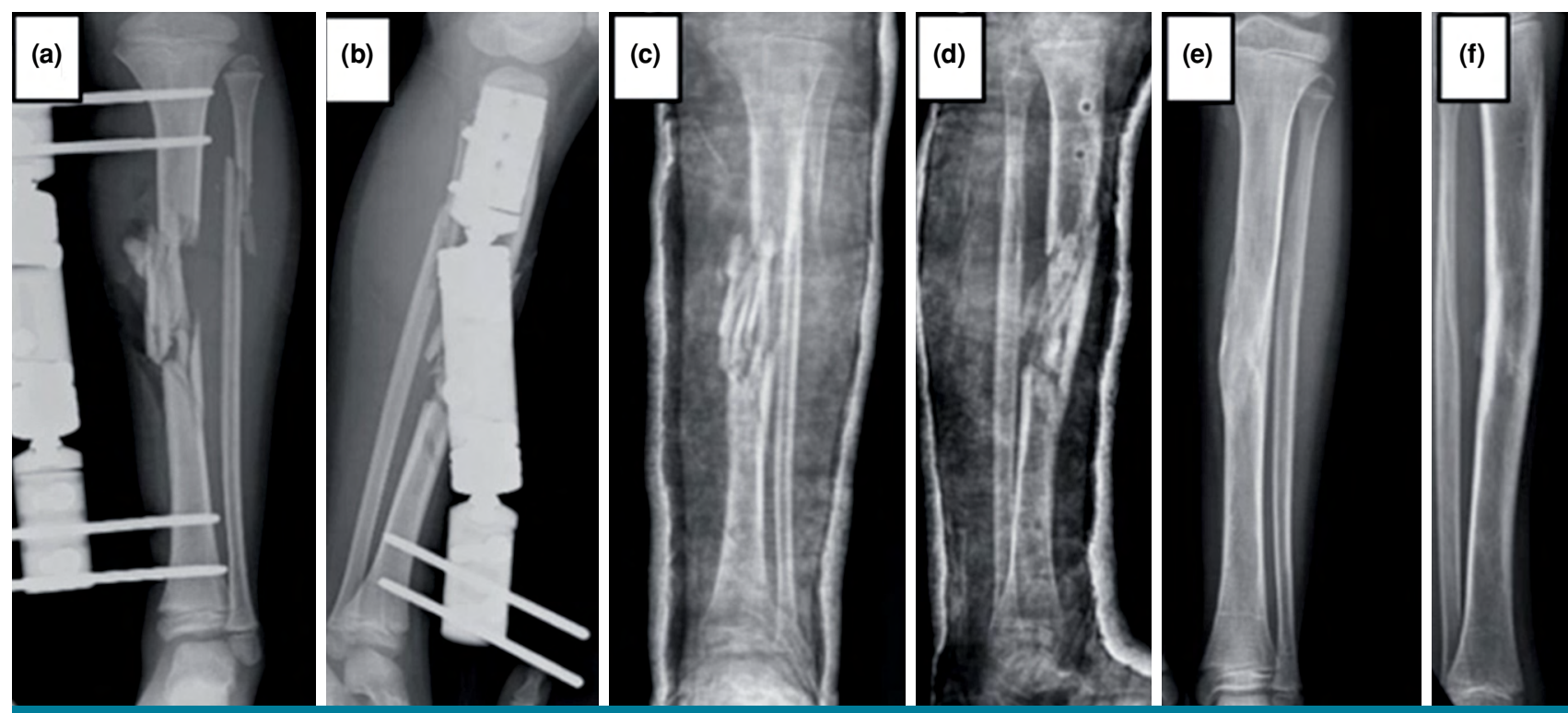

FIGURE 4. An eight-year-old male patient's left tibial and associated fibular fracture and Gustilo Anderson classification type 2 injury after a motor vehicle accident (AO-PCCF 42-D/4.2). (a) First postoperative anteroposterior X-ray. (b) First postoperative lateral view. (c) Anteroposterior view after in situ casting at fourth week. (d) Lateral view after in situ casting at fourth week. (e) First-year anteroposterior radiography, perfect union score in two views, no malalignment after remodeling. (f) First-year lateral radiography, perfect union score in two views, no malalignment after remodeling.

AO-PCCF 42-D/4.2: Arbeitsgemeinschaft für Osteosynthesefragen Pediatric Comprehensive Classification of Long Bone Fractures type 42-D/4.2.

than females (Fisher's exact test, $\mathrm{p}=0.014$ ). There was no relationship between sides and union scores (for fourth, sixth and eighth weeks: $\mathrm{p}=0.510 ; \mathrm{p}=0.613$; $\mathrm{p}=0.935$, respectively). There was no relationship between sex and side with the additional problem $(\mathrm{p}=0.743 ; \mathrm{p}=0.768$, respectively) (Table III).
We had 11 different fracture types according to the AO-PCCF and $42 \mathrm{t}-\mathrm{D} / 5.1$ was the most common type with the number of $13(28.3 \%)$. Due to this diversity of differences, a reliable statistical evaluation could not be performed because of the small number of samples included in the groups.
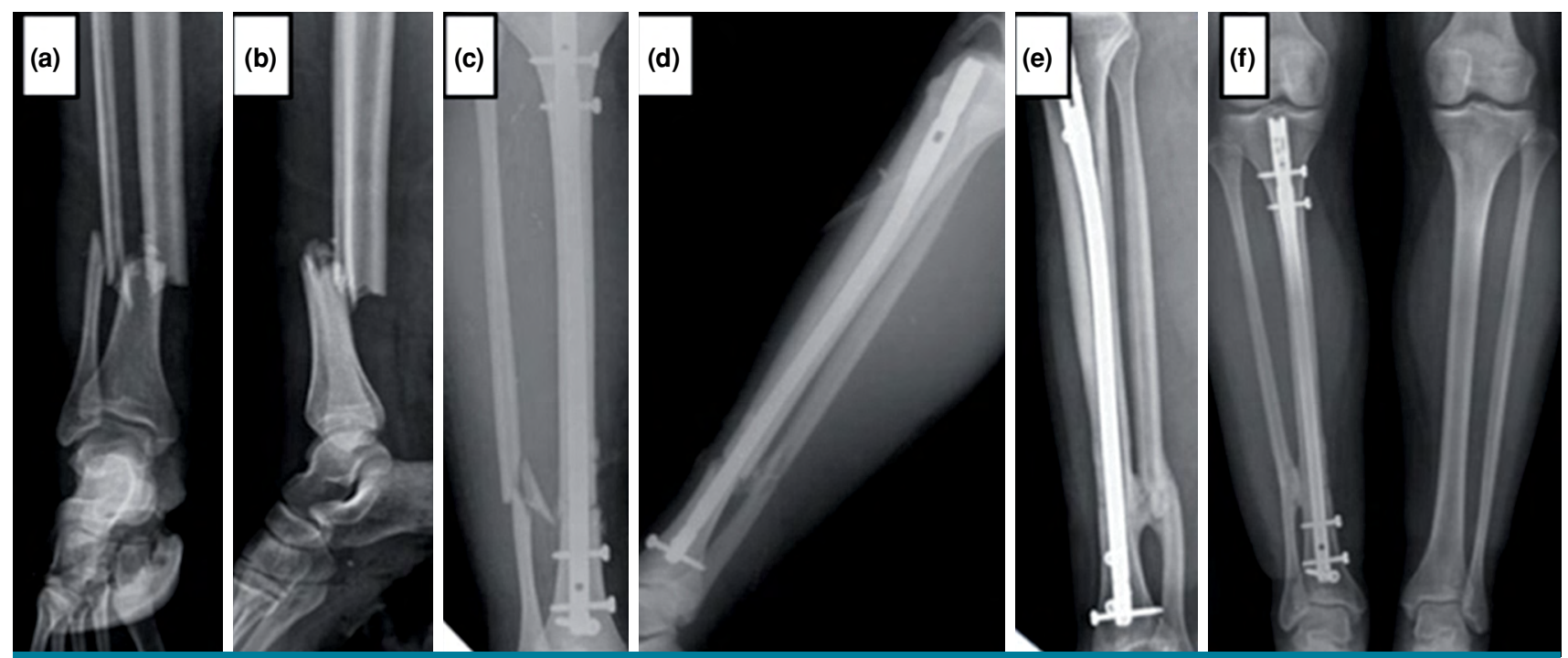

FIGURE 5. A 15-year-old female patient's right tibial and associated fibular fracture after a motor vehicle accident (AO-PCCF 42-D/4.2). (a) Preoperative anteroposterior radiograph. (b) Preoperative lateral view. (c) Anteroposterior view after intramedullary nailing. (d) Lateral view after intramedullary nailing. (e) First-year anteroposterior radiography, perfect union score in two views, synostosis. (f) First-year lateral radiography, perfect union score in two views, synostosis.

AO-PCCF 42-D/4.2: Arbeitsgemeinschaft für Osteosynthesefragen Pediatric Comprehensive Classification of Long Bone Fractures type 42-D/4.2. 


\begin{tabular}{|c|c|c|c|c|}
\hline \multicolumn{5}{|c|}{$\begin{array}{c}\text { TABLE I } \\
\text { Demographic data and informative details about injury }\end{array}$} \\
\hline & & & cription & \\
\hline Parameters & $\mathrm{n}$ & $\%$ & Mean & Range \\
\hline Age (year) & & & 9.5 & $2-16$ \\
\hline $\begin{array}{l}\text { Sex } \\
\quad \text { Males } \\
\text { Females }\end{array}$ & $\begin{array}{l}32 \\
13\end{array}$ & $\begin{array}{l}71.1 \\
28.9\end{array}$ & & \\
\hline $\begin{array}{l}\text { Side } \\
\text { Right } \\
\text { Left } \\
\text { Bilateral }\end{array}$ & $\begin{array}{c}22 \\
22 \\
1\end{array}$ & $\begin{array}{l}48.9 \\
48.9 \\
2.2\end{array}$ & & \\
\hline $\begin{array}{l}\text { Additional injury } \\
\text { Yes } \\
\text { No }\end{array}$ & $\begin{array}{c}4 \\
42\end{array}$ & $\begin{array}{c}8.7 \\
91.3\end{array}$ & & \\
\hline $\begin{array}{l}\text { Fibular fracture } \\
\text { Yes } \\
\text { No }\end{array}$ & $\begin{array}{l}28 \\
18\end{array}$ & $\begin{array}{l}60.9 \\
39.1\end{array}$ & & \\
\hline $\begin{array}{l}\text { Accident } \\
\text { Falling from stairs } \\
\text { Falling from height } \\
\text { Direct trauma } \\
\text { Motor vehicle accident } \\
\text { Falling from the ground level } \\
\text { Farm Injury } \\
\text { Car accident as a } \\
\text { passenger }\end{array}$ & $\begin{array}{c}5 \\
5 \\
5 \\
22 \\
6 \\
2 \\
1\end{array}$ & $\begin{array}{c}10.9 \\
10.9 \\
10.9 \\
47.8 \\
13.0 \\
4.3 \\
2.2\end{array}$ & & \\
\hline $\begin{array}{l}\text { AO fracture type } \\
43 t-M / 3.1 \\
42 t-D / 5.1 \\
43-M / 3.1 \\
42-D / 4.2 \\
42 t-D / 4.2 \\
43-M / 3.2 \\
42-D / 5.1 \\
42-D / 5.2 \\
42-D / 4.1 \\
42 t-D / 4.1 \\
42 t-D / 5.2\end{array}$ & $\begin{array}{c}1 \\
13 \\
5 \\
6 \\
2 \\
2 \\
7 \\
5 \\
3 \\
1 \\
1\end{array}$ & $\begin{array}{c}2.2 \\
28.3 \\
10.9 \\
13.0 \\
4.3 \\
4.3 \\
15.2 \\
10.9 \\
6.5 \\
2.2 \\
2.2\end{array}$ & & \\
\hline $\begin{array}{l}\text { Fracture type } \\
\text { Shaft } \\
\text { Distal metaphysis }\end{array}$ & $\begin{array}{c}37 \\
9\end{array}$ & $\begin{array}{l}80.4 \\
19.6\end{array}$ & & \\
\hline $\begin{array}{l}\text { Gustilo-Anderson } \\
\text { Classification } \\
\text { No open wound } \\
\text { Type 1 } \\
\text { Type 2 } \\
\text { Type 3A } \\
\text { Type 3B } \\
\text { Type 3C }\end{array}$ & $\begin{array}{c}34 \\
2 \\
7 \\
1 \\
0 \\
2\end{array}$ & $\begin{array}{c}73.9 \\
4.3 \\
15.2 \\
2.2 \\
0 \\
4.3\end{array}$ & & \\
\hline $\begin{array}{l}\text { Treatment type } \\
\text { Closed reduction and casting } \\
\text { Closed reduction + pinning } \\
\text { and casting } \\
\text { Intramedullary nail } \\
\text { Titanium elastic nail } \\
\text { Open reduction and plating } \\
\text { External fixator }\end{array}$ & $\begin{array}{l}17 \\
6 \\
1 \\
8 \\
5 \\
9\end{array}$ & $\begin{array}{c}37 \\
13 \\
2.2 \\
17.4 \\
10.9 \\
19.6\end{array}$ & & \\
\hline $\begin{array}{l}\text { Additional operation } \\
\text { Yes } \\
\text { No }\end{array}$ & $\begin{array}{c}4 \\
42\end{array}$ & $\begin{array}{c}8.7 \\
91.3\end{array}$ & & \\
\hline $\begin{array}{l}\text { Additional problem } \\
\text { Yes } \\
\text { No }\end{array}$ & $\begin{array}{l}23 \\
23\end{array}$ & $\begin{array}{l}50 \\
50\end{array}$ & & \\
\hline
\end{tabular}

Therefore, we divided all fractures into two groups according to the level of the fracture: shaft and distal. When we divided the fracture types as shaft and distal, we could not determine any statistically significant difference of union scores for the fourth, sixth and eighth weeks (Mann-Whitney $U$ test, $p=0.191 ; p=0.210 ; p=0.221$, respectively). Also, there was no relationship between fracture level (shaft or distal) and additional problem (Fisher's exact test, $\mathrm{p}=1.000$ ) (Table III).

The number and percentages of the applied treatments are listed in Table I. There was a heterogeneous distribution of treatment types. We divided treatment types into two groups as CRC and operative groups. We had 17 fractures in CRC group (37\%) and 29 fractures in operative group (63\%). The mean age of the 17 patients in CRC group was 7.1 years and the mean age of the 28 patients in operative group was 11.0 years $(p=0.002)$. The union scores of each week were higher in CRC group than operative group ( $\mathrm{p}<0.001 ; \mathrm{p}<0.001 ; \mathrm{p}<0.001$, respectively). There was a negative correlation between age and the union scores $(\mathrm{p}<0.001 / \mathrm{r}=-0.778 ; \mathrm{p}<0.001 / \mathrm{r}=-0.770 ; \mathrm{p}<0.001 /$ $\mathrm{r}=-0.758$, respectively for each week). Three out of 29 operated tibial fractures and one out of 17 patients who were treated by CRC had re-operation $(\mathrm{p}=1.000)$.

\begin{tabular}{|lccc|}
\multicolumn{4}{c}{ TABLE II } \\
Union scores for each week \\
\hline Time & Number & Mean \pm SD & Min-Max \\
\hline $4^{\text {th }}$ Week & 46 & $7.24 \pm 1.99$ & $4.00-12.00$ \\
$6^{\text {th }}$ Week & 45 & $8.91 \pm 2.07$ & $4.00-12.00$ \\
$8^{\text {th }}$ Week & 45 & $10.18 \pm 1.87$ & $4.00-12.00$ \\
\hline SD: Standard deviation; Min: Minimum; Max: Maximum. \\
\hline
\end{tabular}

\begin{tabular}{|c|c|}
\hline \multicolumn{2}{|l|}{$\begin{array}{c}\text { TABLE III } \\
\text { Statistical comparison of parameters w }\end{array}$} \\
\hline & $\begin{array}{l}\text { Comparison with } \\
\text { additional problem } \\
\text { (p) }\end{array}$ \\
\hline Sex & $0.743^{*}$ \\
\hline Side & $0.768^{*}$ \\
\hline Fracture level (shaft-distal) & $1.000^{* *}$ \\
\hline Wound type (open-closed) & $0.007^{*}$ \\
\hline Associated fibular fracture (yes/no) & $0.003^{*}$ \\
\hline $\begin{array}{l}\text { Treatment type (operation or closed } \\
\text { reduction-casting) }\end{array}$ & $<0.001^{*}$ \\
\hline
\end{tabular}




\begin{tabular}{|llcccc}
\multicolumn{7}{c}{ TABLE IV } \\
\multicolumn{7}{c}{ Relationship between union scores and wound condition } & \\
\hline Time & Wound condition & Number & Mean \pm SD & Min-Max & $p^{*}$ \\
\hline $4^{\text {th }}$ Week & Closed & 34 & $7.8 \pm 1.9$ & $4.00-12.00$ & 0.001 \\
& Open & 12 & $5.7 \pm 1.4$ & $4.00-8.00$ & \\
$6^{\text {th }}$ Week & Closed & 33 & $9.6 \pm 1.8$ & $6.00-12.00$ & \\
& Open & 12 & $7.1 \pm 1.7$ & $4.00-10.00$ & $<0.001$ \\
$8^{\text {th }}$ Week & Closed & 33 & $10.8 \pm 1.5$ & $7.00-12.00$ & \\
& Open & 12 & $8.6 \pm 2.0$ & $4.00-11.00$ & 0.001 \\
\hline SD: Standard deviation; Min: Minimum; Max: Maximum; ${ }^{*}$ Mann-Whitney U test. & & \\
\hline
\end{tabular}

\begin{tabular}{|c|c|c|c|c|c|}
\hline \multicolumn{6}{|c|}{$\begin{array}{c}\text { TABLE V } \\
\text { ion scores anc }\end{array}$} \\
\hline Time & Fibular fracture & Number & Mean $\pm S D$ & Min-Max & $p^{*}$ \\
\hline \multirow[t]{2}{*}{$4^{\text {th }}$ Week } & No & 18 & $8.06 \pm 1.98$ & $4.00-11.00$ & \multirow{2}{*}{0.018} \\
\hline & Yes & 28 & $6.71 \pm 1.80$ & $4.00-12.00$ & \\
\hline \multirow[t]{2}{*}{$6^{\text {th }}$ Week } & No & 18 & $9.89 \pm 1.88$ & $6.00-12.00$ & \multirow{2}{*}{0.010} \\
\hline & Yes & 27 & $8.26 \pm 1.95$ & $4.00-12.00$ & \\
\hline \multirow[t]{2}{*}{$8^{\text {th }}$ Week } & No & 18 & $10.94 \pm 1.39$ & $8.00-12.00$ & \multirow{2}{*}{0.017} \\
\hline & Yes & 27 & $9.67 \pm 2.00$ & $4.00-12.00$ & \\
\hline
\end{tabular}

All fractures were classified according to GA classification. Twelve out of 46 fractures had an open wound. The distribution according to GA classification is listed in Table I. The number of sample sizes for groups was not suitable for statistical evaluation. Thus, we classified fractures into two groups as open and closed. We had 34 closed and 12 open fractures. The RUST value of closed group was higher than the open group for fourth, sixth and eighth weeks ( $\mathrm{p}=0.001 ; \mathrm{p}<0.001 ; \mathrm{p}=0.001$, respectively) (Table IV). There was a statistically significant relationship between wound condition and the additional problems ( $\mathrm{p}=0.007)$ (Table III). But no relationship was determined between wound type and additional operation (Fisher's exact test, $\mathrm{p}=0.276$ ).

Eight of nine $(88.9 \%)$ distal metaphyseal tibial fractures and 20 of 37 (54\%) tibial shaft fractures had accompanying fibular fractures (Fischer's exact test, $p=0.069$ ). We observed that the incidence of associated fibular fractures accelerated by growing $(p=0.028)$. The group that did not have associated fibular fractures had better and statistically significantly higher RUST value at fourth, sixth and eighth weeks ( $\mathrm{p}=0.018$; $\mathrm{p}=0.010 ; \mathrm{p}=0.017$, respectively) (Table IV). There was a significant relationship between associated fibular fracture and the 'additional problem' like delayed union after eighth week (Table V). But there was no relationship between fibular fracture and additional operation (Fisher's exact test, $\mathrm{p}=1.000$ ). We had no varus deformity in any patient, no even in the intact fibula group.

\section{DISCUSSION}

Closed reduction and casting is the main treatment of pediatric tibial shaft fractures. As surgical interventions are getting more popular in the treatment of pediatric long bone fractures, casting has become a forgotten and lost art. ${ }^{[4,14,15]}$ We applied CRC only for $37 \%$ of the patients. That means $63 \%$ of the patients had surgery due to their tibial fractures. This rate is higher than the literature ${ }^{[11,14]}$ while the reason for this may be related to having challenging cases in the trauma center because of urban circumstances and not including certain types of fractures such as Toddler's fracture, greenstick and torus fractures which are usually treated conservatively. Twelve out of $46(26.1 \%)$ fractures had associated open wound injury and 16 of 46 were multi-fragmentary fractures; this may be the reason for the high rate of surgery in the present study.

As the child grows, the required force to break the tibia increases. ${ }^{[5]}$ The type of accident changes 
from ground level rotational injury to traffic accident by growing and the incidence of fibular fracture accompanying to the tibial shaft fracture changes according to the type and the severity of injury. Due to the high rate of tibial fracture involvement in abused children, it has to be kept in mind while examining and questioning the family and the child. ${ }^{[3]}$ Only six patients had fallen from the ground level, meaning milder injury compared to falling from stairs, falling from a height, direct trauma, motor vehicle accident (auto, pedestrian or bicycle), farm injury, or car accident as a passenger. All patients could mobilize by themselves (aged two years or higher) and we could not determine any abused children. The mean age of the 22 patients injured by motor vehicle accident was 11.2 years and this was higher than the average of all patients. Nine of the 12 fractures (nine of 11 patients) had motor vehicle accident and this finding is the other indirect indicator of the evidence of the severity of trauma. There is an interesting paradox that has to be taken into consideration: "As the age increases, the mean union score gets worse and the severity of the trauma increases". This means that it is important to be alert while treating adolescent tibial fractures and despite the lack of enough evidence, adult fracture management protocols can be applied for such adolescents. ${ }^{[12,14]}$

The majority of PTFs includes shaft and distal metaphyseal parts (80-90\%). ${ }^{[3,16]}$ Accordingly, our study investigated the most commonly fractured part of the tibia. We had eleven different AO-PCCF type fractures and a heterogeneous distribution. To establish a reliable statistical evaluation, we accepted two different fracture types according to the level of the fracture: shaft and distal. When these two groups were compared according to union score and the additional problem, no significant difference was found in any week.

Different success rates of CRC treatment have been reported in the literature and even in adolescents..$^{[3-5,12,14,16]}$ We had 17 patients with 17 tibial fractures in the CRC group. Only one of those patients who was 16 years old needed operation due to delayed union. This was an expected complication for an adolescent aged 16 years. Sixteen patients obtained the perfect healing score in a minimum of 12-month follow-up. The operated group had three reoperations: one for valgus malalignment, one for bone defect and one for delayed union. This difference in the reoperation was not significant while the difference in the rates between the groups may be related to the different injury types and distribution of the ages. The same paradox mentioned above complicates the situation again. Treatment choice had a significant difference: As the age increases, the usage rate of the CRC decreases (Mann-Whitney $U$ test, $p=0.002$ ). For this reason, it is not suitable to standardize the effect of treatment type without randomized controlled trials with sufficient number of patients in the same conditions.

The open wound injury may lead to delayed union of PTF. ${ }^{[4,17]}$ Hope and Cole ${ }^{[18]}$ reported $16 \%$ delayed union and 7.5\% non-union in their studies with 95 open PTFs. In the present study, we had 12 open fractures and only two of them (16.6\%) required an additional operation due to delayed union. No other case needed any intervention regarding delayed union. Despite the differences in the identification of the delayed union, the rate of reoperation required for delayed union in the present study was similar to that reported by Hope and Cole. ${ }^{[18]}$ The relationship between wound type (open or closed) and postoperative problems ( $p=0.007)$ has to be an attention-increasing parameter while treating pediatric tibial shaft and distal metaphyseal fractures with open wounds.

Accompanying fibular fracture is one of the challenges in treating tibial fractures. The presence of fibular fractures may lead to valgus deformity and the absence may lead to varus deformity. The association rate increases by growing, meaning that the rotational type injuries, which are mostly seen at younger ages, usually have intact fibula. Different rates for accompanying fibular fractures to the tibial shaft fractures were reported in the literature, listed up to $75 \% .^{[2,3,4,11]}$ We had $20(54 \%)$ fibular fractures accompanying to tibial shaft fractures. This rate was higher in the distal metaphyseal group $(88.9 \%)$ while no significant difference was found between the groups ( $p=0.069$ ). The high rate of associated fibular fractures may be due to not including the Toddler's, torus and greenstick fractures in the present study. Although we had $46 \%$ of isolated tibial shaft fractures, we had no varus deformity in any of the patients. However, there were four valgus deformities which all had associated fibular fractures like reported in the literature. ${ }^{[19]}$ Although not statistically significant, the rate of associated fibular fractures was higher in the distal metaphyseal fractures and this may lead healing with valgus deformity.

This study has some limitations. Firstly, the fracture type, GA classification type, age and applied treatments have to be standardized. Secondly, the number of each sample should be sufficient for a more reliable statistical evaluation. Thirdly, we did not include the entire parts of the tibia. These may be both the limitations and also the advantages of this study. 
We need further studies with larger sample sizes and randomized controlled trials to determine the cutoff values for the selection of the treatment type.

In conclusion, associated fibular fractures, open fractures and aging negatively affect union scores of PTF. Attention should be paid, particularly in these conditions, during the selection of the operation type and the follow-up period.

\section{Acknowledgments}

Special thanks to Dr. Deniz Özel Erkan and Akdeniz University Statistical Advisory Unit.

\section{Declaration of conflicting interests}

The authors declared no conflicts of interest with respect to the authorship and/or publication of this article.

\section{Funding}

The authors received no financial support for the research and/or authorship of this article.

\section{REFERENCES}

1. Danseco ER, Miller TR, Spicer RS. Incidence and costs of 1987-1994 childhood injuries: demographic breakdowns. Pediatrics 2000;105:E27.

2. Uludağ A, Tosun HB. Treatment of Unstable Pediatric Tibial Shaft Fractures with Titanium Elastic Nails. Medicina (Kaunas) 2019;55:266.

3. Shannak AO. Tibial fractures in children: follow-up study. J Pediatr Orthop 1988;8:306-10.

4. Mashru RP, Herman MJ, Pizzutillo PD. Tibial shaft fractures in children and adolescents. J Am Acad Orthop Surg 2005;13:345-52.

5. England SP, Sundberg S. Management of common pediatric fractures. Pediatr Clin North Am 1996;43:991-1012.

6. Andreacchio A, Alberghina F, Marengo L, Canavese F. Pediatric tibia and femur fractures in patients weighing more than $50 \mathrm{~kg}(110 \mathrm{lb})$ : mini-review on current treatment options and outcome. Musculoskelet Surg 2019;103:23-30.
7. Rickert KD, Hosseinzadeh P, Edmonds EW. What's new in pediatric orthopaedic trauma: the lower extremity. J Pediatr Orthop 2018;38:e434-e9.

8. Hubbard EW, Riccio AI. Pediatric orthopedic trauma: An evidence-based approach. Orthop Clin North Am 2018;49:195-210.

9. Whelan DB, Bhandari M, Stephen D, Kreder H, McKee MD, Zdero R, et al. Development of the radiographic union score for tibial fractures for the assessment of tibial fracture healing after intramedullary fixation. J Trauma 2010;68:629-32.

10. Litrenta J, Tornetta P 3rd, Mehta S, Jones C, O'Toole $\mathrm{RV}$, Bhandari $\mathrm{M}$, et aj. Determination of Radiographic Healing: An Assessment of Consistency Using RUST and Modified RUST in Metadiaphyseal Fractures. J Orthop Trauma 2015;29:516-20.

11. Mooney JF III, Hennrikus WL. Fractures of the Shaft of Tibia. In: Flynn JM, Skaggs DL, Waters PM, editors. Rockwood and Wilkin's Fractures in Childen. Philadelphia: Wolters Kluwer 8th Edition; 2015. p. 1137-71.

12. AO Pediatric Comprehensive Classification of Long Bone Fractures (PCCF). J Orthop Trauma 2018;32 Suppl 1:S117-S40.

13. Gustilo RB, Mendoza RM, Williams DN. Problems in the management of type III (severe) open fractures: a new classification of type III open fractures. J Trauma 1984;24:742-6.

14. Ho CA. Tibia shaft fractures in adolescents: How and when can they be managed successfully with cast treatment? J Pediatr Orthop 2016;36 Suppl 1:S15-8.

15. Ömeroğlu $H$, Cassiano Neves $M$. Tendency towards operative treatment is increasing in children's fractures: results obtained from patient databases, causes, impact of evidence-based medicine. EFORT Open Rev 2020;5:347-53.

16. Ömeroğlu H. Basic principles of fracture treatment in children. Eklem Hastalik Cerrahisi 2018;29:52-7.

17. Silva M, Eagan MJ, Wong MA, Dichter DH, Ebramzadeh E, Zionts LE. A comparison of two approaches for the closed treatment of low-energy tibial fractures in children. J Bone Joint Surg [Am] 2012;94:1853-60.

18. Hope PG, Cole WG. Open fractures of the tibia in children. J Bone Joint Surg [Br] 1992;74:546-53.

19. Raducha JE, Swarup I, Schachne JM, Cruz AI Jr, Fabricant PD. Tibial Shaft Fractures in Children and Adolescents. JBJS Rev 2019;7:e4. 